\title{
Integrable vortex-type equations on the two-sphere
}

\author{
Alexander D. Popov \\ Bogoliubov Laboratory of Theoretical Physics, JINR \\ 141980 Dubna, Moscow Region, Russia \\ Email: popov@theor.jinr.ru
}

\begin{abstract}
We consider the Yang-Mills instanton equations on the four-dimensional manifold $S^{2} \times \Sigma$, where $\Sigma$ is a compact Riemann surface of genus $g>1$ or its covering space $H^{2}=\mathrm{SU}(1,1) / \mathrm{U}(1)$. Introducing a natural ansatz for the gauge potential, we reduce the instanton equations on $S^{2} \times \Sigma$ to vortex-type equations on the sphere $S^{2}$. It is shown that when the scalar curvature of the manifold $S^{2} \times \Sigma$ vanishes, the vortex-type equations are integrable, i.e. can be obtained as compatibility conditions of two linear equations (Lax pair) which are written down explicitly. Thus, the standard methods of integrable systems can be applied for constructing their solutions. However, even if the scalar curvature of $S^{2} \times \Sigma$ does not vanish, the vortex equations are well defined and have solutions for any values of the topological charge $N$. We show that any solution to the vortex equations on $S^{2}$ with a fixed topological charge $N$ corresponds to a Yang-Mills instanton on $S^{2} \times \Sigma$ of charge $(g-1) N$.
\end{abstract}




\section{Introduction and summary}

The Abelian Higgs model on $\mathbb{R} \times \mathbb{R}^{2}$ at critical value of the coupling constant (the Bogomolny regime) admits static vortex solutions on $\mathbb{R}^{2}[1]$ which describe magnetic flux tubes (vortex strings) penetrating a two-dimensional superconductor. Vortices are important objects in modern field theory [2] since it is believed that (electric) vortex strings play an important role in the confinement of quarks. Their stability is ensured by topology [3]. Many results known for the Abelian Higgs model were generalized to Riemann surfaces, noncommutative spaces and to the non-Abelian case (see e.g. [4]-[13] and references therein).

It was shown recently that the vortex equations on a Riemann surface $\Sigma$ of genus $g$ have a Lax pair representation if $g>1$ and do not have it for $g=0,1$ [8]. This was done by using the correspondence between vortices on $\Sigma$ and $\mathrm{SU}(2)$-equivariant ${ }^{1}$ instantons on the four-manifold $\Sigma \times S^{2}$ - the invariance conditions reduce the instanton equations on $\Sigma \times S^{2}$ to vortex equations on $\Sigma$. Existence of a Lax pair for the reduced equations on $\Sigma$ is related with vanishing of scalar curvature of $\Sigma \times S^{2}$ when this manifold becomes [15] a gravitational instanton. The nonexistence of a Lax pair for vortex equations on $S^{2}, T^{2}$ and $\mathbb{R}^{2}$ followed from the fact that the scalar curvature of $\Sigma \times S^{2}$ is non-vanishing for $\Sigma=S^{2}, T^{2}$ and $\mathbb{R}^{2}$.

In this paper, we introduce an ansatz reducing the instanton equations on $M=S^{2} \times \Sigma$ to vortex-type equations not on $\Sigma$ but on $S^{2}$, and show that these equations are the compatibility conditions of two linear equations (Lax pair) if the scalar curvature of $M$ vanishes, similar to the previous $g>1$ cases [8]. Furthermore, the existence of solutions to the reduced equations on $S^{2}$ for any topological charge $N \geq 0$ demands noncompact initial gauge group for Yang-Mills theory on $M$ and compact gauge group of reduced Yang-Mills-Higgs theory on $S^{2}$. This is similar to the case of the Hitchin equations on $S^{2}$ and $T^{2}$ obtainable as reduction of the instanton equations [16] - smooth solutions on $S^{2}$ (and $T^{2}$ ) exist only if one chooses noncompact gauge group ${ }^{2}$ in four dimensions [19].

The organization of this paper is as follows. In section 2 we collect various facts concerning the geometry of the manifold $S^{2} \times H^{2}$, where $H^{2}=\mathrm{SU}(1,1) / \mathrm{U}(1)$ is the unit disk in the complex plane $\mathbb{C}$. Explicit form of metric, Christoffel symbols etc. are written down. Then, in section 3 , we introduce an $\mathrm{SU}(1,1)$-equivariant ansatz which reduces the instanton equations on $S^{2} \times H^{2}$ to Abelian vortex-type equations on $S^{2}$. Solutions to these equations give solutions of the self-dual Yang-Mills equations on $S^{2} \times H^{2}$ with the noncompact gauge group SU(1,1). Section 4 deals with integrability properties of the introduced Abelian vortex equations. Finally, in section 5 and 6 , we generalize results of section 2-4 to the case of non-Abelian vortex-type equations on $S^{2}$ and instantons on manifolds $S^{2} \times \Sigma$ with compact Riemann surfaces $\Sigma$. Bogomolny transformations for the Yang-Mills-Higgs action functional is discussed and a relation between the instanton and vortex topological charges is derived.

\footnotetext{
${ }^{1}$ This means a generalized SU(2)-invariance, i.e. invariance under space-time transformations up to gauge transformations [14].

${ }^{2}$ Yang-Mills fields with noncompact gauge groups were considered in many papers (see e.g. [17, 18] and references therein).
} 
$2 \quad$ Manifold $S^{2} \times H^{2}$

Riemann sphere. Consider the standard two-sphere $S^{2} \cong \mathbb{C} P^{1}=\mathrm{SU}(2) / \mathrm{U}(1)$ of constant radius $R_{1}$. In local coordinates $y=x^{1}+\mathrm{i} x^{2}, \bar{y}=x^{1}-\mathrm{i} x^{2}$ on $\mathbb{C} P^{1}$ the metric and the volume form read

$$
\mathrm{d} s_{S^{2}}^{2}=2 g_{y \bar{y}} \mathrm{~d} y \mathrm{~d} \bar{y}=\frac{4 R_{1}^{4}}{\left(R_{1}^{2}+y \bar{y}\right)^{2}} \mathrm{~d} y \mathrm{~d} \bar{y}
$$

and

$$
\omega_{S^{2}}=\frac{2 \mathrm{i} R_{1}^{4}}{\left(R_{1}^{2}+y \bar{y}\right)^{2}} \mathrm{~d} y \wedge \mathrm{d} \bar{y}=\mathrm{i} g_{y \bar{y}} \mathrm{~d} y \wedge \mathrm{d} \bar{y},
$$

respectively. For the nonvanishing components of the Christoffel symbols and the Ricci tensor we have

$$
\begin{gathered}
\Gamma_{y y}^{y}=2 \partial_{y} \log \rho_{1} \quad \text { and } \quad \Gamma_{\bar{y} \bar{y}}^{\bar{y}}=2 \partial_{\bar{y}} \log \rho_{1} \quad \text { with } \rho_{1}^{2}:=g_{y \bar{y}} \\
R_{y \bar{y}}=-2 \partial_{y} \partial_{\bar{y}} \log \rho_{1}=\frac{1}{R_{1}^{2}} g_{y \bar{y}} \quad \Longrightarrow \quad R_{S^{2}}=2 g^{y \bar{y}} R_{y \bar{y}}=\frac{2}{R_{1}^{2}}
\end{gathered}
$$

where $R_{S^{2}}$ is the scalar curvature of $S^{2}$.

For the components $g_{y \bar{y}}$ and $g^{y \bar{y}}=1 / g_{y \bar{y}}$ we have

$$
g_{y \bar{y}}=e_{y}^{1} e_{\bar{y}}^{\overline{1}} \quad \text { and } \quad g^{y \bar{y}}=e_{1}^{y} e_{\overline{1}}^{\bar{y}}
$$

where $e_{1}^{y}$ and $e_{\overline{1}}^{\bar{y}}$ are unitary (local) frame. We introduce a basis of type $(1,0)$ and $(0,1)$ vector fields

$$
e_{1}:=e_{1}^{y} \partial_{y} \quad \text { and } \quad e_{\overline{1}}:=e_{\overline{1}}^{\bar{y}} \partial_{\bar{y}}
$$

on $S^{2} \cong \mathbb{C} P^{1}$. The dual basis of type $(1,0)$ and $(0,1)$ forms is $e_{y}^{1} \mathrm{~d} y$ and $e_{\bar{y}}^{\overline{1}} \mathrm{~d} \bar{y}$.

Coset space $H^{2}$. Consider the symmetric space (unit disk)

$$
H^{2}=\mathrm{SU}(1,1) / \mathrm{U}(1)
$$

where $\mathrm{SU}(1,1)$ is a noncompact real form of the group $\mathrm{SL}(2, \mathbb{C})$ with elements $h$ defined by

$$
h^{\dagger} \eta h=\eta \quad \text { for } \quad \eta=\left(\begin{array}{cc}
1 & 0 \\
0 & -1
\end{array}\right)
$$

The metric and the Kähler form in the coordinates $z=x^{3}-\mathrm{i} x^{4}, \bar{z}=x^{3}+\mathrm{i} x^{4}$ on $H^{2}$ are given by

$$
\mathrm{d} s_{H^{2}}^{2}=2 g_{z \bar{z}} \mathrm{~d} z \mathrm{~d} \bar{z}=\frac{4 R_{2}^{4}}{\left(R_{2}^{2}-z \bar{z}\right)^{2}} \mathrm{~d} z \mathrm{~d} \bar{z}
$$

and

$$
\omega_{H^{2}}=-\frac{2 \mathrm{i} R_{2}^{4}}{\left(R_{2}^{2}-z \bar{z}\right)^{2}} \mathrm{~d} z \wedge \mathrm{d} \bar{z}=-\mathrm{i} \beta \wedge \bar{\beta},
$$

where

$$
\beta:=\frac{\sqrt{2} R_{2}^{2} \mathrm{~d} z}{R_{2}^{2}-z \bar{z}} \quad \text { and } \quad \bar{\beta}:=\frac{\sqrt{2} R_{2}^{2} \mathrm{~d} \bar{z}}{R_{2}^{2}-z \bar{z}}
$$


are forms on $H^{2}$ of type $(1,0)$ and $(0,1)$. These forms satisfy the equations

$$
\mathrm{d} \beta=-2 a \wedge \beta, \quad \mathrm{d} \bar{\beta}=2 a \wedge \bar{\beta} \quad \text { and } \quad \mathrm{d} a=-\frac{1}{2 R_{2}^{2}} \beta \wedge \bar{\beta}=-\frac{\mathrm{i}}{2 R_{2}^{2}} \omega_{H^{2}} .
$$

The anti-hermitian connection one-form

$$
a=\frac{1}{2\left(R_{2}^{2}-z \bar{z}\right)}(\bar{z} \mathrm{~d} z-z \mathrm{~d} \bar{z})
$$

with the curvature form $\mathrm{d} a$ given in $(2.12)$ is an $H^{2}$-analog of the monopole connection on $\mathbb{C} P^{1}$. Note that $2 a$ is the Levi-Civita connection on the tangent bundle $T H^{2}$. The one-form $a$ is a connection on the square root $L$ of the holomorphic bundle $T^{1,0} H^{2}$.

The Christoffel symbols, Ricci tensor and scalar curvature for $H^{2}$ are

$$
\begin{aligned}
& \Gamma_{z z}^{z}=2 \partial_{z} \log \rho_{2} \quad \text { and } \quad \Gamma_{\bar{z} \bar{z}}^{\bar{z}}=2 \partial_{\bar{z}} \log \rho_{2} \quad \text { with } \quad \rho_{2}^{2}:=g_{z \bar{z}} \\
& R_{z \bar{z}}=-2 \partial_{z} \partial_{\bar{z}} \log \rho_{2}=-\frac{1}{R_{2}^{2}} g_{z \bar{z}} \quad \Longrightarrow \quad R_{H^{2}}=2 g^{z \bar{z}} R_{z \bar{z}}=-\frac{2}{R_{2}^{2}} .
\end{aligned}
$$

For $(1,0)$ and $(0,1)$ vector fields on $H^{2}$ dual to forms $(2.11)$ we have

$$
e_{2}:=e_{2}^{z} \partial_{z}=\rho_{2}^{-1} \partial_{z} \quad \text { and } \quad e_{\overline{2}}:=e_{\overline{2}}^{\bar{z}} \partial_{\bar{z}}=\rho_{2}^{-1} \partial_{\bar{z}}
$$

with $\rho_{2}$ given in (2.14) and (2.9).

We also consider a four-manifold $M$ given by a product of $S^{2}$ and $H^{2}$ with the product metric

$$
\mathrm{d} s_{M}^{2}=\mathrm{d} s_{S^{2}}^{2}+\mathrm{d} s_{H^{2}}^{2} .
$$

For the scalar curvature of $M=S^{2} \times H^{2}$ we have

$$
R_{M}=R_{S^{2}}+R_{H^{2}}=2\left(\frac{1}{R_{1}^{2}}-\frac{1}{R_{2}^{2}}\right)
$$

\section{Vortices on $S^{2}$ as Yang-Mills configurations on $S^{2} \times H^{2}$}

SU(1,1)-equivariant gauge potential. Consider the manifold $M=S^{2} \times H^{2}$. Let $\mathcal{E} \rightarrow M$ be an $\mathrm{SU}(1,1)$-equivariant complex vector bundle of rank 2 over $M$ with the group $\mathrm{SU}(1,1)$ acting trivially on $S^{2}$ and in the standard way by $\mathrm{SU}(1,1)$-isometry on $H^{2}=\mathrm{SU}(1,1) / \mathrm{U}(1)$. Let $\mathcal{A}$ be an $s u(1,1)$-valued local form of $\mathrm{SU}(1,1)$-equivariant connection on $\mathcal{E}$ (cf. [7, 8]); it can be chosen in the form

$$
\mathcal{A}=\left(\begin{array}{cc}
\frac{1}{2} A \otimes 1+1 \otimes a & \frac{1}{\sqrt{2}} \phi \otimes \beta \\
\frac{1}{\sqrt{2}} \bar{\phi} \otimes \bar{\beta} & -\frac{1}{2} A \otimes 1-1 \otimes a
\end{array}\right)=\left(\frac{1}{2} A+a\right) \sigma_{3}+\frac{1}{\sqrt{2}} \phi \beta \sigma_{+}+\frac{1}{\sqrt{2}} \bar{\phi} \bar{\beta} \sigma_{-},
$$

where

$$
\sigma_{3}=\left(\begin{array}{cc}
1 & 0 \\
0 & -1
\end{array}\right), \quad \sigma_{+}=\left(\begin{array}{ll}
0 & 1 \\
0 & 0
\end{array}\right) \quad \text { and } \quad \sigma_{-}=\left(\begin{array}{cc}
0 & 0 \\
1 & 0
\end{array}\right)
$$


Here, $A=A_{y} \mathrm{~d} y+A_{\bar{y}} \mathrm{~d} \bar{y}$ is an Abelian connection on a (Hermitian) complex line bundle $E$ over $\mathbb{C} P^{1} \cong S^{2}, a$ is the connection (2.13) on the complex line bundle $L$ over $H^{2}, \phi$ is a section of the bundle $E, \bar{\phi}$ is its complex conjugate and forms $\beta, \bar{\beta}$ on $H^{2}$ are given in (2.11). In local complex coordinates $y, \bar{y}$ on $\mathbb{C} P^{1}$ we have $A=A(y, \bar{y})$ and $\phi=\phi(y, \bar{y})$.

Field strength tensor. In local coordinates on $S^{2} \times H^{2}$ the calculation of the curvature $\mathcal{F}$ for $\mathcal{A}$ of the form (3.1) yields

$$
\begin{gathered}
\mathcal{F}=\mathrm{d} \mathcal{A}+\mathcal{A} \wedge \mathcal{A}=\left(\begin{array}{cc}
\frac{1}{2} F-\frac{1}{2}\left(\frac{1}{R_{2}^{2}}-\phi \bar{\phi}\right) \beta \wedge \bar{\beta} & \frac{1}{\sqrt{2}}(\mathrm{~d} \phi+A \phi) \wedge \beta \\
\frac{1}{\sqrt{2}}(\mathrm{~d} \bar{\phi}-A \bar{\phi}) \wedge \bar{\beta} & -\frac{1}{2} F+\frac{1}{2}\left(\frac{1}{R_{2}^{2}}-\phi \bar{\phi}\right) \beta \wedge \bar{\beta}
\end{array}\right) \\
=\mathcal{F}_{y \bar{y}} \mathrm{~d} y \wedge \mathrm{d} \bar{y}+\mathcal{F}_{y z} \mathrm{~d} y \wedge \mathrm{d} z+\mathcal{F}_{y \bar{z}} \mathrm{~d} y \wedge \mathrm{d} \bar{z}+\mathcal{F}_{\bar{y} z} \mathrm{~d} \bar{y} \wedge \mathrm{d} z+\mathcal{F}_{\bar{y} \bar{z}} \mathrm{~d} \bar{y} \wedge \mathrm{d} \bar{z}+\mathcal{F}_{z \bar{z}} \mathrm{~d} z \wedge \mathrm{d} \bar{z}
\end{gathered}
$$

with the non-vanishing field strength components

$$
\begin{gathered}
\mathcal{F}_{y \bar{y}}=\frac{1}{2} F_{y \bar{y}} \sigma_{3}, \quad \mathcal{F}_{z \bar{z}}=-\frac{1}{2} g_{z \bar{z}}\left(\frac{1}{R_{2}^{2}}-\phi \bar{\phi}\right) \sigma_{3}, \\
\mathcal{F}_{\bar{y} z}=\frac{\rho_{2}}{\sqrt{2}}\left(\partial_{\bar{y}} \phi+A_{\bar{y}} \phi\right) \sigma_{+}, \quad \mathcal{F}_{y z}=\frac{\rho_{2}}{\sqrt{2}}\left(\partial_{y} \phi+A_{y} \phi\right) \sigma_{+}, \\
\mathcal{F}_{y \bar{z}}=\frac{\rho_{2}}{\sqrt{2}}\left(\partial_{y} \bar{\phi}-A_{y} \bar{\phi}\right) \sigma_{-}, \quad \mathcal{F}_{\bar{y} \bar{z}}=\frac{\rho_{2}}{\sqrt{2}}\left(\partial_{\bar{y}} \bar{\phi}-A_{\bar{y}} \bar{\phi}\right) \sigma_{-} .
\end{gathered}
$$

In (3.4) we have defined $F=\mathrm{d} A=F_{y \bar{y}} \mathrm{~d} y \wedge \mathrm{d} \bar{y}=\left(\partial_{y} A_{\bar{y}}-\partial_{\bar{y}} A_{y}\right) \mathrm{d} y \wedge \mathrm{d} \bar{y}$ for $A=A_{y} \mathrm{~d} y+A_{\bar{y}} \mathrm{~d} \bar{y}$.

Vortex equations on $S^{2}$. Let us consider the self-dual Yang-Mills equations $* \mathcal{F}=\mathcal{F}$ on $S^{2} \times H^{2}$, where $*$ is the Hodge operator. In local coordinates these equations have the form

$$
\mathcal{F}_{\bar{y} \bar{z}}=0=\left(\mathcal{F}_{y z}\right)^{\dagger} \quad \text { and } \quad g^{y \bar{y}} \mathcal{F}_{y \bar{y}}+g^{z \bar{z}} \mathcal{F}_{z \bar{z}}=0 .
$$

Substitution of (3.4)-(3.6) into (3.7) shows that the self-dual Yang-Mills equations (3.7) on $S^{2} \times H^{2}$ are equivalent to the BPS vortex-type equations on $S^{2}$ :

$$
\begin{gathered}
F_{y \bar{y}}=g_{y \bar{y}}\left(\frac{1}{R_{2}^{2}}-\phi \bar{\phi}\right) \quad \Leftrightarrow \quad \mathrm{i} F=\left(\frac{1}{R_{2}^{2}}-\phi \bar{\phi}\right) \omega_{S^{2}}, \\
\partial_{y} \phi+A_{y} \phi=0 \quad \Leftrightarrow \quad \partial_{A} \phi=0,
\end{gathered}
$$

where $\partial_{A}=\mathrm{d} y\left(\partial_{y}+A_{y}\right)$. Note that for the standard vortex equations instead of eq.(3.9) one has $\partial_{\bar{y}} \phi+A_{\bar{y}} \phi=0$. This equation can be obtained if in (3.1) one choose $\bar{\beta}$ in the upper right corner and $-\beta$ in the lower left corner (compact gauge group $\mathrm{SU}(2)$ ) but then in (3.8) one will have $-1 / R_{2}^{2}$ and such vortex-type equations will not have solutions due to the Kazdan-Warner theorem [20].

Vortex number $N$ is defined as the first Chern number $c_{1}(E)$ of the bundle $E \rightarrow \mathbb{C} P^{1}$,

$$
N=c_{1}(E)=\frac{\mathrm{i}}{2 \pi} \int_{S^{2}} F \text {. }
$$


From (3.8) it follows that

$$
\frac{\mathrm{i}}{2 \pi} \int_{S^{2}} F+\frac{1}{2 \pi} \int_{S^{2}} \phi \bar{\phi} \omega_{S^{2}}=\frac{1}{2 \pi R_{2}^{2}} \int_{S^{2}} \omega_{S^{2}}=2\left(\frac{R_{1}}{R_{2}}\right)^{2},
$$

and we obtain (cf. [4]) the inequality

$$
N \leq 2\left(\frac{R_{1}}{R_{2}}\right)^{2}
$$

For any $N \geq 0$ the condition (3.12) can be satisfied for sufficiently large ratio $R_{1} / R_{2}$ and then the moduli space of vortices on $S^{2}$ will be nonempty.

Liouville-type equations on $S^{2}$. Consider $N$-vortex solution $\phi=\exp \left(\frac{1}{2}(u+\mathrm{i} \theta)\right)$, where $u$ and $\theta$ are real-valued functions. Since $\phi$ can have zeros at $y_{i} \in \mathbb{C} P^{1}$, then $u(y) \rightarrow-\infty$ as $y \rightarrow y_{i}$ and $\theta(y)$ is a multi-valued function with ramification points at $y_{i}$. The equation (3.9) implies that

$$
A_{y}=-\partial_{y} \log \phi=-\frac{1}{2} \partial_{y}(u+\mathrm{i} \theta) \quad \text { and } \quad A_{\bar{y}}=\partial_{\bar{y}} \log \bar{\phi}=\frac{1}{2} \partial_{\bar{y}}(u-\mathrm{i} \theta) .
$$

Plugging (3.13) into (3.8), we obtain the Liouville-type equations on $S^{2}$,

$$
\partial_{y} \partial_{\bar{y}} u=g_{y \bar{y}}\left(\frac{1}{R_{2}^{2}}-e^{u}\right)
$$

away from the singularities of $u$.

Note that the sign on the right hand side of eq.(3.14) with $g_{y \bar{y}}$ given in (2.1) is opposite to the sign in the standard vortex equations on $S^{2}$. However, equations of type (3.14) on a compact Riemann surfaces (including $S^{2}$ ) were considered by Kazdan and Warner [20]. They have shown, in particular, that equations

$$
\partial_{y} \partial_{\bar{y}} u= \pm g_{y \bar{y}}\left(\frac{1}{R_{2}^{2}}-e^{u}\right)
$$

have solutions for both signs in (3.15) and equations

$$
\partial_{y} \partial_{\bar{y}} u=\mp g_{y \bar{y}}\left(\frac{1}{R_{2}^{2}}+e^{u}\right)
$$

have no solutions. These four cases exhaust possible Liouville-type equations on $S^{2}$ with $R_{2}^{2} \neq \infty$.

Recall that equations (3.15) can be obtained by the reduction of the self-dual Yang-Mills (SDYM) equations from $S^{2} \times S^{2}$ to $S^{2}$ with gauge group $\mathrm{SU}(2)$ (lower sign) and from $S^{2} \times H^{2}$ to $S^{2}$ with gauge group SU(1,1) (upper sign). Similarly, equations (3.16) correspond to the reduction of the SDYM equations from $S^{2} \times H^{2}$ to $S^{2}$ with gauge group $\mathrm{SU}(2)$ (lower sign) and from $S^{2} \times S^{2}$ to $S^{2}$ with gauge group $\mathrm{SU}(1,1)$ (upper sign). Thus, only the gauge group $\mathrm{SU}(1,1)$ is allowed for the considered case of the reduction $S^{2} \times H^{2} \rightarrow S^{2}$, and solutions of (3.14) exist for any $N \geq 0$.

If one considers the reduction of the SDYM equations from $S^{2} \times H^{2}$ to $H^{2}$, the allowed gauge group is $\mathrm{SU}(2)$ [8]. In other words, depending on a symmetry ( $\mathrm{SU}(2)$ - or $\mathrm{SU}(1,1)$-equivariance) imposed on gauge fields, on $S^{2} \times H^{2}$ there exist solutions of the SDYM equations with gauge groups as $\mathrm{SU}(2)$ and $\mathrm{SU}(1,1)$. 


\section{Integrability of vortex equations on $S^{2}$}

Integrable case. We considered the BPS vortex-type equations (3.8)-(3.9) and showed their equivalence to the self-dual Yang-Mills equations (3.7) on the manifold $M=S^{2} \times H^{2}$. Note that for equal radii $R_{1}=R_{2}$ of $S^{2}$ and $H^{2}$ the scalar curvature (2.18) of $M$ vanishes. In this case the Weyl tensor for the manifold $M$ is self-dual [15].

An important feature of Kähler manifolds $M$ with scalar curvature $R_{M}$ is that the so-called twistor space $\mathcal{Z}$ of $M$ becomes a complex manifold if $R_{M}=0$. Let us consider an open subset $\mathcal{U}$ of $M=S^{2} \times H^{2}$ with complex coordinates $y, z$. Then the twistor space of $\mathcal{U}$ (i.e. the restriction of $\mathcal{Z}$ to $\mathcal{U})$ is diffeomorphic to $\mathcal{U} \times \mathbb{C} P^{1},\left.\mathcal{Z}\right|_{\mathcal{U}} \simeq \mathcal{U} \times \mathbb{C} P^{1}$, with a local complex coordinate $\lambda \in \mathbb{C} P^{1} \backslash\{\infty\}$ on the last factor. On $\mathcal{Z}$ there is a distribution generated by three vector fields of type $(0,1)$ closed under the Lie bracket. They have the form (cf. [8])

$$
V_{\overline{1}}:=\tilde{e}_{\overline{1}}-\lambda \tilde{e}_{2}, \quad V_{\overline{2}}:=\tilde{e}_{\overline{2}}+\lambda \tilde{e}_{1} \quad \text { and } \quad V_{\overline{3}}=\partial_{\bar{\lambda}}
$$

where

$$
\begin{array}{ll}
\tilde{e}_{1}=\rho_{1}^{-1}\left(\partial_{y}-\left(\partial_{y} \log \rho_{1}\right) \lambda \partial_{\lambda}\right), & \tilde{e}_{\overline{1}}=\rho_{1}^{-1}\left(\partial_{\bar{y}}+\left(\partial_{\bar{y}} \log \rho_{1}\right) \lambda \partial_{\lambda}\right), \\
\tilde{e}_{2}=\rho_{2}^{-1}\left(\partial_{z}-\left(\partial_{z} \log \rho_{2}\right) \lambda \partial_{\lambda}\right), & \tilde{e}_{\overline{2}}=\rho_{2}^{-1}\left(\partial_{\bar{z}}+\left(\partial_{\bar{z}} \log \rho_{2}\right) \lambda \partial_{\lambda}\right) .
\end{array}
$$

Recall that $\rho_{1}^{2}=g_{y \bar{y}}$ and $\rho_{2}^{2}=g_{z \bar{z}}$ are components of metrics on $S^{2}$ and $H^{2}$; their explicit forms are given in section 2 .

The vector fields (4.1) define an almost complex structure $\mathcal{J}$ on $\mathcal{Z}$ such that

$$
\mathcal{J}\left(V_{\bar{k}}\right)=-\mathrm{i} V_{\bar{k}}
$$

for $k=1,2,3$. For commutators of type $(0,1)$ vector fields $(4.1)$ we have

$$
\left[V_{\overline{1}}, V_{\overline{2}}\right]=\lambda \rho_{1}^{-2}\left(\partial_{y} \rho_{1}\right) V_{\overline{1}}+\lambda \rho_{2}^{-2}\left(\partial_{z} \rho_{2}\right) V_{\overline{2}}+2 \lambda^{2}\left(\frac{1}{R_{1}^{2}}-\frac{1}{R_{2}^{2}}\right) V_{3}, \quad\left[V_{\overline{1}}, V_{\overline{3}}\right]=0=\left[V_{\overline{2}}, V_{\overline{3}}\right],
$$

where $V_{3}=\partial_{\lambda}$ is the $(1,0)$ vector field on $\mathcal{Z}$. Recall that for integrability of an almost complex structure $\mathcal{J}$ on $\mathcal{Z}$ it is necessary and sufficient that the commutator of any two vector fields of type $(0,1)$ w.r.t. $\mathcal{J}$ is of type $(0,1)$. For our case we see from (4.5) that $\mathcal{J}$ is integrable - and $\mathcal{Z}$ is a complex manifold - if and only if

$$
R_{1}=R_{2}
$$

i.e when the scalar curvature $R_{M}$ of the manifold $M=S^{2} \times H^{2}$ vanishes. In this case the bundle $\mathcal{E} \rightarrow M$ pulled back to the bundle $\hat{\mathcal{E}}$ over the twistor space $\mathcal{Z}$ allows an integrable holomorphic structure defined by a $(0,1)$-type connection along the vector fields (4.1). The integrability of this structure, $\mathcal{F}^{0,2}=0$, is equivalent [21] to the self-duality equations on $M$.

Lax pair. For the case (4.6) from (3.12) we obtain the inequality

$$
N \leq 2
$$

i.e. bundles $\hat{\mathcal{E}}$ over $\mathcal{Z}$ with integrable holomorphic structures describe configurations of $N=1$ and $N=2$ vortices on $S^{2}$. We emphasize that vortices exist for any $N>0$ but only for $N \leq 2$ the vortex equations (3.8)-(3.9) appear from a Lax pair. 
For presenting vortex equations on $S^{2}$ as an integrable system (for $R_{1}=R_{2}$ ) one should introduce two linear equations (Lax pair) whose compatibility conditions will produce the vortex equations. For that we introduce a $(0,1)$ part $\hat{\nabla}^{0,1}$ of the covariant derivative $\hat{\nabla}$ on $\hat{\mathcal{E}}$ by formulae

$$
\begin{aligned}
\hat{\nabla}_{V_{\overline{1}}} & \equiv V_{\overline{1}}+\hat{\mathcal{A}}_{V_{\overline{1}}}:=\tilde{e}_{\overline{1}}-\lambda \tilde{e}_{2}+\mathcal{A}_{\overline{1}}-\lambda \mathcal{A}_{2}, \\
\hat{\nabla}_{V_{\overline{2}}} & \equiv V_{\overline{2}}+\hat{\mathcal{A}}_{V_{\overline{2}}}:=\tilde{e}_{\overline{2}}+\lambda \tilde{e}_{1}+\mathcal{A}_{\overline{2}}+\lambda \mathcal{A}_{1}, \\
\hat{\nabla}_{V_{\overline{3}}} & \equiv V_{\overline{3}}+\hat{\mathcal{A}}_{V_{\overline{3}}}:=\partial_{\bar{\lambda}},
\end{aligned}
$$

where $\hat{\nabla}_{X}$ denotes the covariant derivative along the vector field $X$. The components

$$
\mathcal{A}_{1}=e_{1}^{y} \mathcal{A}_{y}, \quad \mathcal{A}_{\overline{1}}=e_{\overline{1}}^{\bar{y}} \mathcal{A}_{\bar{y}}, \quad \mathcal{A}_{2}=e_{2}^{z} \mathcal{A}_{z} \quad \text { and } \quad \mathcal{A}_{\overline{2}}=e_{\overline{2}}^{\bar{z}} \mathcal{A}_{\bar{z}}
$$

are easily extracted from (3.1).

Let us now introduce a $2 \times 2$ matrix $\psi=\psi(y, \bar{y}, z, \bar{z}, \lambda)$ which does not depend on $\bar{\lambda}$ and consider two linear equations

$$
\begin{aligned}
\hat{\nabla}_{V_{\overline{1}}} \psi & :=\left[\tilde{e}_{\overline{1}}+\mathcal{A}_{\overline{1}}-\lambda\left(\tilde{e}_{2}+\mathcal{A}_{2}\right)\right] \psi=0, \\
\hat{\nabla}_{V_{\overline{2}}} \psi & \left.:=\left[\lambda\left(\tilde{e}_{1}+\mathcal{A}_{1}\right)+\tilde{e}_{\overline{2}}+\mathcal{A}_{\overline{2}}\right)\right] \psi=0 .
\end{aligned}
$$

It is not difficult to check that the compatibility conditions of the linear equations (4.10),

$$
\left(\left[\hat{\nabla}_{V_{\overline{1}}}, \hat{\nabla}_{V_{\overline{2}}}\right]-\hat{\nabla}_{\left[V_{\overline{1}}, V_{\overline{2}}\right]}\right) \psi=0
$$

are equivalent to the vortex equations (3.8)-(3.9) for $\mathcal{A}$ given in (3.1).

Note that equations

$$
\hat{\mathcal{F}}^{0,2}=0 \quad \Leftrightarrow \quad \hat{\mathcal{F}}\left(V_{\bar{\imath}}, V_{\bar{\jmath}}\right)=\left[\hat{\nabla}_{V_{\bar{\imath}}}, \hat{\nabla}_{V_{\bar{j}}}\right]-\hat{\nabla}_{\left[V_{\bar{\imath}}, V_{\bar{\jmath}}\right]}=0,
$$

for $\hat{\nabla}_{V_{\bar{J}}}$ given in the first two formulae from (4.8) can be imposed even if an almost complex structure $\mathcal{J}$ on $\mathcal{Z}$ is not integrable, that is, the case when $R_{1} \neq R_{2}$. Then equations (4.12) define a pseudo-holomorphic structure $[22]$ on the bundle $\hat{\mathcal{E}} \rightarrow \mathcal{Z}$. These equations are again equivalent to the self-duality equations on $S^{2} \times H^{2}$ since

$$
\hat{\mathcal{F}}\left(V_{\overline{1}}, V_{\overline{2}}\right)=\mathcal{F}_{\overline{1} \overline{2}}-\lambda\left(\mathcal{F}_{1 \overline{1}}+\mathcal{F}_{2 \overline{2}}\right)+\lambda^{2} \mathcal{F}_{12}, \quad \hat{\mathcal{F}}\left(V_{\overline{1}}, V_{\overline{3}}\right)=0=\hat{\mathcal{F}}\left(V_{\overline{2}}, V_{\overline{3}}\right),
$$

where

$$
\begin{aligned}
& \mathcal{F}_{\overline{1} \overline{2}}=e_{\overline{1}} \mathcal{A}_{\overline{2}}-e_{\overline{2}} \mathcal{A}_{\overline{1}}+\left[\mathcal{A}_{\overline{1}}, \mathcal{A}_{\overline{2}}\right]=e_{\overline{1}}^{\bar{y}} e_{\overline{2}}^{\bar{z}} \mathcal{F}_{\bar{y} \bar{z}}, \\
& \mathcal{F}_{12}=e_{1} \mathcal{A}_{2}-e_{2} \mathcal{A}_{1}+\left[\mathcal{A}_{1}, \mathcal{A}_{2}\right]=e_{1}^{y} e_{2}^{z} \mathcal{F}_{y z} \\
& \mathcal{F}_{1 \overline{1}}=e_{1} \mathcal{A}_{\overline{1}}-e_{\overline{1}} \mathcal{A}_{1}+\left[\mathcal{A}_{1}, \mathcal{A}_{\overline{1}}\right]-\rho_{1}^{-1}\left(e_{\overline{1}} \rho_{1}\right) \mathcal{A}_{1}+\rho_{1}^{-1}\left(e_{1} \rho_{1}\right) \mathcal{A}_{\overline{1}}=g^{y \bar{y}} \mathcal{F}_{y \bar{y}}, \\
& \mathcal{F}_{2 \overline{2}}=e_{2} \mathcal{A}_{\overline{2}}-e_{\overline{2}} \mathcal{A}_{2}+\left[\mathcal{A}_{2}, \mathcal{A}_{\overline{2}}\right]-\rho_{2}^{-1}\left(e_{\overline{2}} \rho_{2}\right) \mathcal{A}_{2}+\rho_{2}^{-1}\left(e_{2} \rho_{2}\right) \mathcal{A}_{\overline{2}}=g^{z \bar{z}} \mathcal{F}_{z \bar{z}}
\end{aligned}
$$

After substituting SU(1,1)-equivariant gauge potential (3.1), eqs. (4.13) reduce to the vortex equations on $S^{2}$ having solutions with $N>2$. So, for $N>2$ vortex equations on $S^{2}$ do not appear as a compatibility condition of a Lax pair but are derivable nevertheless from the self-dual YangMills equations similarly to vortex equations on Riemann surfaces with genus $g>1$, where vortex equations were integrable only for $N \leq 2(g-1)$ [8]. 


\section{Quiver vortex equations}

Here and in section 6, we generalize the equations (3.8)-(3.9) to the non-Abelian case and describe relations between vortices on $S^{2}$ and instantons on the manifold $S^{2} \times \Sigma$, where $\Sigma$ is a compact Riemann surface of genus $g>1$.

Equivariant vector bundle. Consider the manifold $M=\mathbb{C} P^{1} \times H^{2}$, where $\mathbb{C} P^{1} \cong S^{2}$ is the Riemann sphere and $H^{2}$ is the unit disk described in section 2 . Let $\mathcal{E} \rightarrow M$ be an $\mathrm{SU}(1,1)$ equivariant rank- $k$ complex vector bundle, with the group $\mathrm{SU}(1,1)$ acting trivially on $\mathbb{C} P^{1}$ and by isometry on $H^{2}=\mathrm{SU}(1,1) / \mathrm{U}(1)$. Let $\mathcal{A}$ be a connection on $\mathcal{E}$. Imposing the condition of $\mathrm{SU}(1,1)$ equivariance means that we should look for representations of the group $\mathrm{SU}(1,1)$ on $\mathbb{C}^{k}$. Notice that for each positive integer $m$, the module

$$
\mathbb{C}^{k}=\bigoplus_{i=0}^{m} \mathbb{C}^{k_{i}} \quad \text { with } \quad \sum_{i=0}^{m} k_{i}=k
$$

gives such a representation if $\mathbb{C}^{m+1}$ is an irreducible representation of $\mathrm{SU}(1,1)$. Let

$$
E=\bigoplus_{i=0}^{m} E_{i} \rightarrow \mathbb{C} P^{1}
$$

be a rank- $k \mathbb{Z}_{m+1}$-graded complex vector bundle over $\mathbb{C} P^{1}$ and $A^{i}$ 's are connection forms on the bundles $E_{i} \rightarrow \mathbb{C} P^{1}$. Then

$$
\mathcal{E}=\bigoplus_{i=0}^{m} \mathcal{E}_{i} \quad \text { with } \quad \mathcal{E}_{i}=E_{i} \otimes L^{m-2 i}
$$

where $L^{m-2 i}=(L)^{\otimes(m-2 i)}$ and the bundle $L \rightarrow H^{2}$ with a connection $a$ given in (2.13) was introduced in section 2 .

Symmetric gauge potential and field strength tensor. Similar to the compact $\mathrm{SU}(2)$ case [7], the $\mathrm{SU}(1,1)$-equivariant gauge potential $\mathcal{A}$ with values in End $\mathbb{C}^{k}$ decomposes into connections $A^{i} \in u\left(k_{i}\right)$ on the complex rank- $k_{i}$ vector bundles $E_{i} \rightarrow \mathbb{C} P^{1}$ with $i=0,1, \ldots, m$ and a multiplet of scalar fields $\phi_{i+1}$ on $\mathbb{C} P^{1}$ with $i=0,1, \ldots, m-1$ transforming in the bi-fundamental representation $\mathbb{C}^{k_{i}} \otimes\left(\mathbb{C}^{\vee}\right)^{k_{i}+1}$ of the group $\mathrm{U}\left(k_{i}\right) \times \mathrm{U}\left(k_{i+1}\right)$, i.e. $\phi \in \operatorname{Hom}\left(E_{i}, E_{i+1}\right)$. Collecting these Higgs fields into the upper triangular $k \times k$ complex matrix

$$
\phi_{(m)}:=\left(\begin{array}{ccccc}
0 & \phi_{1} & 0 & \ldots & 0 \\
0 & 0 & \phi_{2} & \ldots & 0 \\
\vdots & \vdots & \ddots & \ddots & \vdots \\
0 & 0 & 0 & \ldots & \phi_{m} \\
0 & 0 & 0 & \ldots & 0
\end{array}\right),
$$

we get

$$
\mathcal{A}=A^{(m)} \otimes 1+\Upsilon_{(m)} \otimes a+\frac{1}{\sqrt{2}} \phi_{(m)} \otimes \beta+\frac{1}{\sqrt{2}} \phi_{(m)}^{\dagger} \otimes \bar{\beta}
$$

where

$$
A^{(m)}:=\sum_{i=0}^{m} A^{i} \otimes \Pi_{i}, \quad \Upsilon_{(m)}:=\sum_{i=0}^{m}(m-2 i) 1_{k_{i}} \otimes \Pi_{i}
$$


and $\Pi_{i}: E \rightarrow E_{i}$ are the canonical orthogonal projectors of rank $1, \Pi_{i} \Pi_{j}=\delta_{i j} \Pi_{j}$, which may be represented by diagonal $(m+1) \times(m+1)$ matrices $\Pi_{i}=\left(\delta_{j i} \delta_{l i}\right)_{j, l=0,1, \ldots, m}$ of unit trace. Here $\beta$ and $\bar{\beta}$ are forms on $H^{2}$ of type $(1,0)$ and $(0,1)$ defined in section 2 .

The calculation of the curvature $\mathcal{F}$ for $\mathcal{A}$ of the form (5.5) yields

$$
\begin{gathered}
\mathcal{F}=\mathrm{d} \mathcal{A}+\mathcal{A} \wedge \mathcal{A}=F^{(m)} \otimes 1-\frac{1}{2}\left(\frac{1}{R_{2}^{2}} \Upsilon_{(m)}-\left[\phi_{(m)}, \phi_{(m)}^{\dagger}\right]\right) \beta \wedge \bar{\beta} \\
+\frac{1}{\sqrt{2}}\left(\mathrm{~d} \phi_{(m)}+\left[A^{(m)}, \phi_{(m)}\right]\right) \wedge \beta+\frac{1}{\sqrt{2}}\left(\mathrm{~d} \phi_{(m)}^{\dagger}+\left[A^{(m)}, \phi_{(m)}^{\dagger}\right]\right) \wedge \bar{\beta},
\end{gathered}
$$

where $F^{(m)}=\mathrm{d} A^{(m)}+\left[A^{(m)}, A^{(m)}\right]$. The derivation of (5.7) uses formulae (2.12).

Non-Abelian vortex equations on $\mathbb{C} P^{1}$. Let us consider the self-dual Yang-Mills equations $* \mathcal{F}=\mathcal{F}$ on $M$. In local coordinates on $M$ these equations have the form (3.7). Substitution of (5.7) into the instanton equations on $M=S^{2} \times H^{2}$ reduce them to non-Abelian quiver vortex equations (cf. [7]-[8])

$$
\begin{gathered}
\text { i } F^{(m)}=\frac{1}{2}\left(\frac{1}{R^{2}} \Upsilon_{(m)}-\left[\phi_{(m)}, \phi_{(m)}^{\dagger}\right]\right) \omega_{S^{2}}, \\
\partial \phi_{(m)}+\left[A_{(1,0)}^{(m)}, \phi_{(m)}\right]=0,
\end{gathered}
$$

where $\partial=\mathrm{d} y \partial_{y}$ and $\omega_{S^{2}}$ is given in (2.2). In terms of $\left(A^{i}, \phi_{i}\right)$ these equations have the form

$$
\begin{gathered}
2 \mathrm{i} F^{i}=\left(\frac{m-2 \mathrm{i}}{R^{2}} 1_{k_{i}}+\phi_{i}^{\dagger} \phi_{i}-\phi_{i+1} \phi_{i+1}^{\dagger}\right) \omega_{S^{2}}, \\
\partial \phi_{i+1}+A_{(1,0)}^{i} \phi_{i+1}-\phi_{i+1} A_{(1,0)}^{i+1}=0,
\end{gathered}
$$

where $A_{(1,0)}^{i}=A_{y}^{i} \mathrm{~d} y, i=0, \ldots, m$ and $\phi_{0}:=0=: \phi_{m+1}$. Finally, in local complex coordinates on $\mathbb{C} P^{1}$ we get

$$
\begin{gathered}
2 g^{y \bar{y}} F_{y \bar{y}}^{i}=\frac{m-2 \mathrm{i}}{R^{2}} 1_{k_{i}}+\phi_{i}^{\dagger} \phi_{i}-\phi_{i+1} \phi_{i+1}^{\dagger}, \\
\partial_{y} \phi_{i+1}+A_{y}^{i} \phi_{i+1}-\phi_{i+1} A_{y}^{i+1}=0
\end{gathered}
$$

\section{Instantons with noncompact gauge groups}

Riemann surfaces. Recall that any simply connected Riemann surface $\Sigma$ of genus $g>1$ is conformally equivalent to the unit disk $H^{2}=\mathrm{SU}(1,1) / \mathrm{U}(1)$. In other words, $H^{2}$ is a universal cover of $\Sigma=H^{2} / \Gamma$, where $\Gamma$ is a Fuchsian group ${ }^{3}$. Here, we will show that the ansatz (5.5) can also be used on the manifold $M=S^{2} \times \Sigma$, where $\Sigma$ is a compact Riemann surface of genus $g>1$.

The metric and the volume form on $\Sigma$ in local (conformal) coordinates $z, \bar{z}$ are given by

$$
\mathrm{d} s_{\Sigma}^{2}=2 g_{z \bar{z}} \mathrm{~d} z \mathrm{~d} \bar{z} \quad \text { and } \quad \omega_{\Sigma}=\mathrm{i} g_{z \bar{z}} \mathrm{~d} z \wedge \mathrm{d} \bar{z}
$$

\footnotetext{
${ }^{3}$ It is a discrete subgroup of the group $\mathrm{SU}(1,1) \cong \mathrm{SL}(2, \mathbb{R})$
} 
Furthermore, for the nonvanishing components of the Christoffel symbols and the Ricci tensor we have

$$
\begin{aligned}
& \Gamma_{z z}^{z}=2 \partial_{z} \log \rho \quad \text { and } \Gamma_{\bar{z} \bar{z}}^{\bar{z}}=2 \partial_{\bar{z}} \log \rho \quad \text { with } \rho^{2}:=g_{z \bar{z}}, \\
& R_{z \bar{z}}=-2 \partial_{z} \partial_{\bar{z}} \log \rho=\varkappa g_{z \bar{z}} \quad \Longrightarrow \quad R_{\Sigma}=2 g^{z \bar{z}} R_{z \bar{z}}=2 \varkappa,
\end{aligned}
$$

where $R_{\Sigma}$ is the constant scalar curvature of $\Sigma$. The area of the Riemann surface with genus $g \neq 1$ is

$$
\operatorname{Vol}(\Sigma)=\int_{\Sigma} \omega_{\Sigma}=\frac{4 \pi}{\varkappa}(1-g) .
$$

Introducing forms $\beta$ and $\bar{\beta}$ of type $(1,0)$ and $(0,1)$ on $\Sigma$,

$$
\beta:=\rho \mathrm{d} z \quad \text { and } \quad \bar{\beta}:=\rho \mathrm{d} \bar{z} \quad \Longrightarrow \quad \mathrm{d} s_{\Sigma}^{2}=2 \beta \bar{\beta},
$$

we obtain that

$$
\mathrm{d} \beta=-2 a \wedge \beta, \quad \mathrm{d} \bar{\beta}=2 a \wedge \bar{\beta} \quad \text { and } \quad \mathrm{d} a=\frac{1}{2} \varkappa \beta \wedge \bar{\beta},
$$

where

$$
2 a=\left(\partial_{z} \log \rho\right) \mathrm{d} z-\left(\partial_{\bar{z}} \log \rho\right) \mathrm{d} \bar{z}
$$

is the Levi-Civita $u(1)$-connection on the tangent bundle $T \Sigma$ of $\Sigma$. Denoting the holomorphic part $T^{1,0} \Sigma$ of $T \Sigma \otimes \mathbb{C}$ by $L^{2}$, we obtain the complex line bundle $L \rightarrow \Sigma$ with the connection $a$. Finally, after choosing

$$
\varkappa=-\frac{1}{R^{2}},
$$

we see that $a, \beta$ and $\bar{\beta}$ in (6.6) satisfy the same equations as forms in (2.12) and therefore the ansatz (5.5) on the manifold $\mathbb{C} P^{1} \times \Sigma$ yields to the curvature (5.7) and to the quiver vortex equations (5.8)-(5.13). That is why, in what follows we will consider our gauge theory on the compact spaces $M=\mathbb{C} P^{1} \times \Sigma$.

Reduction of the Yang-Mills functional. The dimensional reduction of the Yang-Mills equations from $\mathbb{C} P^{1} \times \Sigma$ to $\mathbb{C} P^{1}$ can also be seen at the level of the Yang-Mills Lagrangian. For simplicity, we consider the case $m=1$ for which the instanton equations on $\mathbb{C} P^{1} \times \Sigma$ are equivalent to the equations (3.8)-(3.9) with $A=2 A^{0}, \phi=\phi_{1}$ and $R=R_{2}$. Substituting (5.5)-(5.7) with $m=1$ into the standard Yang-Mills functional and performing the integral over $\Sigma$, we arrive at the action

$$
\begin{aligned}
S & =-\frac{1}{8 \pi^{2}} \int_{S^{2} \times \Sigma} \operatorname{tr}(\mathcal{F} \wedge * \mathcal{F})=-\frac{1}{16 \pi^{2}} \int_{S^{2} \times \Sigma} \mathrm{d}^{4} x \sqrt{\operatorname{det}\left(g_{\rho \sigma}\right)} \operatorname{tr}\left(\mathcal{F}_{\mu \nu} \mathcal{F}^{\mu \nu}\right) \\
& =(g-1) \frac{R^{2}}{4 \pi} \int_{S^{2}} \omega_{S^{2}}\left\{\left(g^{y \bar{y}}\right)^{2}\left(F_{y \bar{y}}\right)^{2}-2 g^{y \bar{y}}\left(D_{y} \phi \overline{D_{y} \phi}+D_{\bar{y}} \phi \overline{D_{\bar{y}} \phi}\right)+\left(\frac{1}{R^{2}}-\phi \bar{\phi}\right)^{2}\right\} \\
& =(g-1) \frac{R^{2}}{4 \pi} \int_{S^{2}} \mathrm{i} \mathrm{d} y \wedge \mathrm{d} \bar{y}\left\{g^{y \bar{y}}\left(F_{y \bar{y}}+g^{y \bar{y}}\left(\phi \bar{\phi}-\frac{1}{R^{2}}\right)\right)^{2}-4 D_{y} \phi \overline{D_{y} \phi}\right\}+(g-1) \frac{\mathrm{i}}{2 \pi} \int_{S^{2}} F,
\end{aligned}
$$

where $\mu, \nu, \ldots=1, \ldots, 4, D_{y}=\partial_{y}+A_{y}$ and $D_{\bar{y}}=\partial_{\bar{y}}+A_{\bar{y}}$. On solutions $(A, \phi)$ of vortex equations (3.8)-(3.9) this action coincides with $(g-1) N$, where

$$
N=\frac{\mathrm{i}}{2 \pi} \int_{S^{2}} F=c_{1}(E)
$$


is the vortex number.

Topological charges. For self-dual gauge fields we have

$$
N_{\text {inst }}=-c_{2}(\mathcal{E})=-\frac{1}{8 \pi^{2}} \int_{S^{2} \times \Sigma} \operatorname{tr}(\mathcal{F} \wedge \mathcal{F})=(g-1) \frac{\mathrm{i}}{2 \pi} \int_{S^{2}} F=(g-1) N,
$$

i.e. the instanton number $N_{\text {inst }}$ is proportional to the vortex number $N$. In the derivation of (6.9)(6.11) it is assumed that $N \geq 0 .{ }^{4}$ From (6.9) we see that due to noncompactness of the gauge group $\mathrm{SU}(1,1)$ the energy density for vortices is not positive definite but for $(A, \phi)$ satisfying the BPS vortex equations (3.8)-(3.9) the action $S$ coincides with the topological invariant $(g-1) c_{1}(E)=$ $-c_{2}(\mathcal{E})$. Thus, by solving equations $(5.8)-(5.9)$ on $\mathbb{C} P^{1}$ one can obtain instantons on $\mathbb{C} P^{1} \times \Sigma$ with noncompact gauge group and the topological charge $N_{\text {inst }}=(g-1) N$.

Acknowledgments. I would like to thank Olaf Lechtenfeld for useful discussions and the Institute for Theoretical Physics of Leibniz Universität Hannover, where this work was completed, for hospitality. This work was partially supported by the Alexander von Humboldt Foundation.

\section{References}

[1] A.A. Abrikosov, "On the magnetic properties of superconductors of the second group," Sov. Phys. JETP 5 (1957) 1174 [Zh. Eksp. Teor. Fiz. 32 (1957) 1442];

H.B. Nielsen and P. Olesen, "Vortex line models for dual strings," Nucl. Phys. B 61 (1973) 45.

[2] N. Manton and P. Sutcliffe, Topological solitons, Cambridge University Press, Cambridge, 2004.

[3] A. Jaffe and C. Taubes, Vortices and monopoles, Birkhäuser, Boston, 1980.

[4] S.B. Bradlow, "Vortices in holomorphic line bundles over closed Kähler manifolds," Commun. Math. Phys. 135 (1990) 1;

O. García-Prada, "A direct existence proof for the vortex equations over a compact Riemann surface." Bull. London Math. Soc. 26 (1994) 88.

[5] N.S. Manton, "Statistical mechanics of vortices," Nucl. Phys. B 400 (1993) 624;

P.A. Shah and N.S. Manton, "Thermodynamics of vortices in the plane,"

J. Math. Phys. 35 (1994) 1171 [hep-th/9307165].

[6] N.S. Manton and S.M. Nasir, "Volume of vortex moduli spaces,"

Commun. Math. Phys. 199 (1999) 591 [hep-th/9807017];

J.M. Baptista and N.S. Manton, "The dynamics of vortices on $S^{2}$ near the Bradlow limit," J. Math. Phys. 44 (2003) 3495 [hep-th/0208001].

\footnotetext{
${ }^{4}$ For $N \leq 0$ one should consider the anti-self-dual Yang-Mills equations $* \mathcal{F}=-\mathcal{F}$ which reduce to anti-vortex equations.
} 
[7] A.D. Popov and R.J. Szabo, "Quiver gauge theory of nonabelian vortices and noncommutative instantons in higher dimensions," J. Math. Phys. 47 (2006) 012306 [hep-th/0504025];

O. Lechtenfeld, A.D. Popov and R.J. Szabo, "Rank two quiver gauge theory, graded connections and noncommutative vortices," JHEP 09 (2006) 054 [hep-th/0603232];

O. Lechtenfeld, A.D. Popov and R.J. Szabo, "Quiver gauge theory and noncommutative vortices," Prog. Theor. Phys. Suppl. 171 (2007) 258 [arXiv:0706.0979 [hep-th]].

[8] A.D. Popov, "Integrability of vortex equations on Riemann surfaces,"

Nucl. Phys. B 821 (2009) 452 [arXiv:0712.1756 [hep-th]];

A.D. Popov, "Non-Abelian vortices on Riemann surfaces: an integrable case,"

Lett. Math. Phys. 84 (2008) 139 [arXiv:0801.0808 [hep-th]].

[9] M. Eto, Y. Isozumi, M. Nitta, K. Ohashi and N. Sakai, "Solitons in the Higgs phase: the moduli matrix approach," J. Phys. A 39 (2006) R315 [hep-th/0602170];

T. Fujimori, M. Nitta, K. Ohta, N. Sakai and M. Yamazaki, "Intersecting solitons, amoeba and tropical geometry," Phys. Rev. D 78 (2008) 105004 [arXiv:0805.1194 [hep-th]];

T. Fujimori, G. Marmorini, M. Nitta, K. Ohashi and N. Sakai,

"The moduli space metric for well-separated non-Abelian vortices,"

Phys. Rev. D 82 (2010) 065005 [arXiv:1002.4580 [hep-th]];

M. Eto, T. Fujimori, M. Nitta, K. Ohashi and N. Sakai, "Dynamics of non-Abelian vortices," Phys. Rev. D 84 (2011) 125030 [arXiv:1105.1547 [hep-th]].

[10] H. Ikemori, S. Kitakado, H. Otsu and T. Sato, "Non-Abelian vortices as instantons on noncommutative discrete space," JHEP 02 (2009) 004 [arXiv:0808.2396 [hep-th]];

H. Otsu, T. Sato, H. Ikemori and S. Kitakado, "Vortices as instantons in noncommutative discrete space: use of Z(2) coordinates,"

Mod. Phys. Lett. A 25 (2010) 2189 [arXiv:0904.1848 [hep-th]];

T. Kimura and M. Nitta, "Vortices on orbifolds," JHEP 09 (2011) 118 [arXiv:1108.3563 [hep-th]];

T. Fujimori, T. Kimura, M. Nitta and K. Ohashi, "Vortex counting from field theory," JHEP 06 (2012) 028 [arXiv:1204.1968 [hep-th]];

M. Eto, T. Fujimori, M. Nitta and K. Ohashi, "All exact solutions of non-Abelian vortices from Yang-Mills instantons," arXiv:1207.5143 [hep-th].

[11] D. Harland and S. Kurkcuoglu, "Equivariant reduction of Yang-Mills theory over the fuzzy sphere and the emergent vortices," Nucl. Phys. B 821 (2009) 380 [arXiv:0905.2338 [hep-th]];

G. Landi and R.J. Szabo, "Dimensional reduction over the quantum sphere and non-Abelian Q-vortices," Commun. Math. Phys. 308 (2011) 365 [arXiv:1003.2100 [hep-th]];

S. Kurkcuoglu, "Noncommutative vortices and flux-tubes from Yang-Mills theories with spontaneously generated fuzzy extra dimensions,"

Phys. Rev. D 82 (2010) 105010 [arXiv:1009.1880 [hep-th]];

S. Kurkcuoglu, "Equivariant reduction of U(4) gauge theory over $S_{F}^{2} \times S_{F}^{2}$ and the emergent vortices," Phys. Rev. D 85 (2012) 105004 [arXiv:1201.0728 [hep-th]].

[12] O. Lechtenfeld, A.D. Popov and R.J. Szabo, "SU(3)-equivariant quiver gauge theories and non-Abelian vortices," JHEP 08 (2008) 093 [arXiv:0806.2791 [hep-th]];

A.D. Popov, "Non-Abelian vortices, super-Yang-Mills theory and Spin(7)-instantons," 
Lett. Math. Phys. 92 (2010) 253 [arXiv:0908.3055 [hep-th]];

D. Harland and A.D. Popov, "Yang-Mills fields in flux compactifications on homogeneous manifolds with SU(4)-structure," JHEP 02 (2012) 107 [arXiv:1005.2837 [hep-th]];

A.D. Popov and R.J. Szabo, "Double quiver gauge theory and nearly Kähler flux compactifications," JHEP 02 (2012) 033 [arXiv:1009.3208 [hep-th]].

[13] N.S. Manton and N.A. Rink, "Vortices on hyperbolic surfaces,"

J. Phys. A 43 (2010) 434024 [arXiv:0912.2058 [hep-th]];

N.S. Manton and N. Sakai, "Maximally non-Abelian vortices from self-dual Yang-Mills fields," Phys. Lett. B 687 (2010) 395 [arXiv:1001.5236 [hep-th]];

N.S. Manton and N.A. Rink, "Geometry and energy of non-Abelian vortices,"

J. Math. Phys. 52 (2011) 043511 [arXiv:1012.3014 [hep-th]].

[14] P. Forgács and N.S. Manton, "Space-time symmetries in gauge theories,"

Commun. Math. Phys. 72 (1980) 15;

D. Kapetanakis and G. Zoupanos, "Coset space dimensional reduction of gauge theories," Phys. Rept. 219 (1992) 1.

[15] C.P. Boyer, "Conformal duality and compact complex surfaces." Math. Ann. 274 (1986) 517;

C. LeBrun, "On the topology of self-dual 4-manifolds." Proc. Amer. Math. Soc. 98 (1986) 637.

[16] N.J. Hitchin, "The self-duality equations on a Riemann surface,"

Proc. London Math. Soc. 55 (1987) 59.

[17] L. Dolan, "Complex solutions of Yang-Mills," Phys. Lett. B 76 (1978) 105;

C. Rebbi, "Self-dual Yang-mills fields in Minkowski space-time," Phys. Rev. D 17 (1978) 483;

A.D. Burns, "Complex string solutions of the self-dual Yang-Mills equations,"

J. Phys. A 17 (1984) 689.

[18] L. Dallagnol and M. Jardim, "Nonsingular complex instantons on Euclidean spacetime,"

Int. J. Geom. Meth. Mod. Phys. 5 (2008) 963.

[19] R.A. Mosna and M. Jardim, "Nonsingular solutions of Hitchin's equations for noncompact gauge groups," Nonlinearity 20 (2007) 1893 [arXiv: math-ph/0609001].

[20] J.L. Kazdan and F.L. Warner, "Curvature functions for compact 2-manifolds," Ann. Math. 99 (1974) 14.

[21] R.S. Ward, "On self-dual gauge fields," Phys. Lett. A 61 (1977) 81;

M.F. Atiyah, N.J. Hitchin and I.M. Singer, "Self-duality in four-dimensional Riemannian geometry," Proc. Roy. Soc. Lond. A 362 (1978) 425.

[22] R.L. Bryant, "On the geometry of almost complex 6-manifolds,"

Asian J. Math. 10 (2006) 561. 